\title{
NEW DEBRIS DISK CANDIDATES AROUND 49 NEARBY STARS
}

\author{
D. W. Koerner ${ }^{1}$, S. Kim ${ }^{1}$, D. E. Trilling ${ }^{1}$, H. Larson ${ }^{1}$, A. Cotera ${ }^{2}$, K. R. Stapelfeldt ${ }^{3}$, Z. Wahhaj ${ }^{4}$, S. Fajardo-Acosta ${ }^{5}$, \\ D. PADGETT ${ }^{5}$, AND D. BACKMAN ${ }^{6}$ \\ ${ }^{1}$ Department of Physics \& Astronomy, Northern Arizona University, Flagstaff AZ 86011, USA \\ ${ }^{2}$ SETI Institute, 515 N. Whisman Road, Mountain View, CA 94043, USA \\ 3 Jet Propulsion Laboratory, California Institute of Technology, USA \\ ${ }^{4}$ Institute for Astronomy, University of Hawaii, 2680 Woodlawn Drive, Honolulu, HI 96822, USA \\ ${ }^{5}$ Spitzer Science Center, California Institute of Technology, Pasadena, CA 91125, USA \\ ${ }^{6}$ Stratospheric Observatory for Infrared Astronomy (SOFIA)/SETI Institute, Mountain View, CA 94043, USA \\ Received 2009 July 24; accepted 2009 December 8; published 2010 January 20
}

\begin{abstract}
We present 49 new candidate debris disks that were detected around nearby stars with the Spitzer Space Telescope using the Multiband Imaging Photometer (MIPS) at $24 \mu \mathrm{m}$ (MIPS24) and $70 \mu \mathrm{m}$ (MIPS70). The survey sample was composed of stars within 25 pc of the Sun that were not previously observed by any other MIPS survey. Only stars with $V<9$ were selected, corresponding to spectral types earlier than M0. MIPS24 integration times were chosen to detect the stellar photosphere at 10 $\sigma$ levels or better. MIPS70 observations were designed to detect excess infrared emission from any star in the MIPS70 sample with a disk as luminous at that around $\epsilon$ Eridani. The resulting sample included over 436 nearby stars that were observed with both MIPS24 and MIPS70, plus an additional 198 observed only with MIPS24. Debris disk candidates were defined as targets where excess emission was detected at $3 \sigma$ levels or greater, and the ratio of observed flux density to expected photosphere emission was three standard deviations or more above the mean value for the sample. The detection rate implied by the resulting 29 MIPS24 candidates is $4.6 \%$. A detection rate of $4.8 \%$ is implied by 21 MIPS70 candidates. The distribution of spectral types for stars identified as candidates resembles that of the general sample and yields strong evidence that debris-disk occurrence does not decrease for $\mathrm{K}$ dwarfs. Modeling of non-uniform sensitivity in the sample is required to interpret quantitative estimates of the overall detection frequency and will be presented in a future work.
\end{abstract}

Key words: circumstellar matter - infrared: stars - protoplanetary disks - solar neighborhood

\section{INTRODUCTION}

The infrared signature of nearby "debris disks" was first discovered with IRAS and identified with circumstellar dust grains around newly dubbed "Vega-type" stars of largely mainsequence age. Increasing evidence supports the early attribution of excess infrared emission to radiation from dust grains that are generated by collisions within a population of asteroidlike bodies or "planetesimals." The vastly improved sensitivity of the Spitzer Space Telescope has yielded a solid legacy of new disk detections and expanded our knowledge of their frequency of occurrence, especially for later spectral types. Unlike the IRAS all-sky survey, however, targeted surveys were necessary to obtain a complete census of debris disks in the solar neighborhood, and a global picture is just started to emerge from combined small-sample surveys.

Gautier et al. (2007) detected no excesses for stars within $5 \mathrm{pc}$ of the Sun (largely M dwarfs), but Beichman et al. (2005) detected Multiband Imaging Photometer (MIPS) $70 \mu \mathrm{m}$ (MIPS70) excess in $25 \%$ of a small sample of FGK dwarfs known to have one or more planets. Rieke et al. (2005) detected new infrared excesses in a MIPS $24 \mu \mathrm{m}$ (MIPS24) survey of 76 A stars; comparison with stellar ages revealed prominent differences in debris-disk properties among similar-age stars and implied that circumstellar dust masses do not decrease monotonically with time. An expanded study of 160 A stars with both MIPS24 and MIPS70 by Su et al. (2006) yielded detection rates of $32 \%$ and $33 \%$, respectively. Bryden et al. (2006) found a disk detection frequency of $1 \%-2 \%$ at $24 \mu \mathrm{m}$ and $10 \%$ at $70 \mu \mathrm{m}$ for a sample of 69 FGK stars, while Beichman et al. (2006) reported a MIPS70 detection rate of
$13 \% \pm 3 \%$ for a survey of $82 \mathrm{~F}, \mathrm{G}, \mathrm{K}$, and a few $\mathrm{M}$ stars. Weak MIPS24 excesses in four of their targets with MIPS70 detections suggested a correlation. They detected no infrared excess emission in any of 38 targets with spectral types in the range K1-M6 and concluded that Spitzer detection rates decline with later spectral type. A survey of 69 A3-F8 binary systems yielded slightly higher detection rates than singlestar surveys with MIPS24 and MIPS70 (Trilling et al. 2007), suggesting that binary companions do not inhibit planetesimal formation. Observations of over $200 \mathrm{~F}$ and $\mathrm{G}$ main-sequence stars by Trilling et al. (2008) yielded a MIPS24 detection rate of $4.2_{-1.1}^{+2.0} \%$ and $16.4_{-2.9}^{+2.8} \%$ for MIPS70. These seminal results comprise a discrete and sparse sampling of the immediate stellar neighborhood. To complete the picture, we recently carried out MIPS Cycle-3 observations of 634 nearby stars under Program 30490, "Completing the Census of Debris Disks Around Nearby Stars," as described below. Here we report candidate debris disks identified in this survey. Analysis of the complete sample will follow in a much longer manuscript.

\section{OBSERVATIONS AND ANALYSIS}

Our sample consists of all stars in the Hipparcos catalog located within $25 \mathrm{pc}$ of the Sun with $V<9$ (spectral types earlier than M0) and not yet listed in the Spitzer Reserved Object Catalog. These criteria produced a total sample of 634 targets that were observed with MIPS24. MIPS70 observations were carried out for a subset of these that exhibited sufficiently lowbackground emission at $70 \mu \mathrm{m}$. Background emission from cirrus and zodiacal dust at $70 \mu \mathrm{m}$ was estimated using the IRAS Sky Survey as accessed by IRSKY Batch Inquiry System (IBIS; 
Wheelock et al. 1993). A limit of $2 \mathrm{mJy}$ for confusion due to background galaxies was taken from empirical analysis by Bryden et al. (2006). Cirrus and zodiacal background limits from IBIS were combined in quadrature with the background galaxy limit from Bryden et al. (2006) to estimate a total background sensitivity limit for each target. Sources were eliminated from the MIPS70 sample if they required integration times of greater than an hour according to sensitivity criteria described below. Approximately one third of the targets from the initial sample were eliminated in this way, largely due to background noise associated with their location in the galactic plane. The remaining 436 targets were observed with MIPS70, and all 634 were observed with MIPS24.

MIPS24 integration times were chosen to detect photosphere emission at the $10 \sigma$ level. Cirrus emission and background galaxies precluded MIPS70 photosphere detection at that level for many of the more distant later-type sources. We aimed, instead, for sensitivity to disks such as that around the K2V star $\epsilon$ Eridani ( $\epsilon$ Eri). IRAS measurements indicated that $\epsilon$ Eri radiates excess infrared emission at more than three times the photosphere level at $60 \mu \mathrm{m}$ and would have been easily detected at the $25 \mathrm{pc}$ distance of our most-distant targets. MIPS70 integration times were estimated for a $3 \sigma$ detection of an $\epsilon$ Eri-like disk everywhere in our volume as per the IRAS $60 \mu \mathrm{m}$ measurement. Subsequent Spitzer MIPS70 observations of $\epsilon$ Eri revealed a ninefold increase of excess over photosphere emission (Backman et al. 2009). Consequently, our survey probed disks with one third of $\epsilon$ Eri's $70 \mu \mathrm{m}$ excess. Nevertheless, this criterion left many of our faintest sources undetected unless they had significant excess.

MIPS Basic Calibrated Data (BCD) frames were processed with MOPEX software to build Post-BCD mosaic images. Manual aperture photometry was carried out on sky-subtracted images using the Global Astrometric Interferometer for Astrophysics (GAIA) package. Aperture sizes for source emission were $13^{\prime \prime}$ and $16^{\prime \prime}$, respectively, for MIPS24 and MIPS70, and aperture corrections of 1.167 and 1.968 were applied. Background emission was measured in annuli with inner and outer radii of $20^{\prime \prime}$ and $32^{\prime \prime}$ for MIPS24 and with $18^{\prime \prime}$ and $39^{\prime \prime}$ for MIPS70. To identify excess infrared emission, overall flux densities were compared to expected photosphere levels.

The photospheric $24 \mu \mathrm{m}$ flux was predicted by extrapolating from near infrared magnitudes with a $K_{s}-$ [24] color relation that was derived from ground-based photometry and Spitzer observations. $K_{s}$ values were taken from the Two Micron All Sky Survey (2MASS; Skrutskie et al. 1997). For $24 \mu \mathrm{m}$ fluxes, we assumed that the majority of our target stars had no $24 \mu \mathrm{m}$ excess and used our own MIPS24 observations. MIPS24 magnitudes were generated as outlined by Rieke et al. (2008), assuming a zero-point magnitude flux of $7.17 \mathrm{Jy} . K_{s}-$ [24] was then calculated for each target and averaged over all targets within a spectral subtype. $K_{s}-[24]$ values varied from 0.009 to 0.05 over the spectral range, $\mathrm{A} 0$ to $\mathrm{K} 5$, and increased from 0.05 to 0.2 for subtypes between K5 and M0. MIPS70 photosphere values were estimated by extrapolating from the observed MIPS24 flux as described by Trilling et al. (2008).

Nearby or early-type target stars with $K_{s}<4$ were bright enough to exceed the 2MASS saturation limit and had correspondingly greater uncertainties in color-derived photosphere estimates if 2MASS values were used. For these, we used Phoenix/NextGen model fitting to measurements in the literature, including Johnson $B, V, 2 \mathrm{MASS} J, H, K_{s}$ (Hauschildt et al. 1999), and the IRAS $12 \mu \mathrm{m}$ flux. Model fluxes for each Spitzer band were computed by multiplying the Phoenix/ NextGen model with the MIPS filter function before scaling and fitting (Hauschildt et al. 1999; Rieke et al. 2004). SEDFIT software (J. Rhee \& I. Song 2007, private communication) was modified to carry out model fitting and estimate both MIPS24 and MIPS70 photospheric values.

As illustrated in Figures 1 and 2 of Beichman et al. (2005) for MIPS24 and MIPS70, respectively, the distributions of observed-to-predicted photosphere ratios were fitted as a Gaussian function to check the method and extract a conservative estimate of the uncertainty in the photospheric estimation process. Ratios near unity are expected for a sample of stars where very few display excess infrared emission. MIPS24 ratios peaked sharply at 0.99 with $\sigma=0.04$. MIPS70 values peaked at 1.09 with $\sigma=0.28$.

To identify debris-disk candidates, we selected targets with infrared excess at $3 \sigma$ levels or higher, i.e., $\chi_{\lambda}=$ $\left(F_{\lambda}-F \mathrm{phot}_{\lambda}\right) / \sigma_{\lambda}>3$. The uncertainty was calculated as $\sigma_{\lambda}=\sqrt{\left(\sigma_{\lambda, \text { systematic }}\right)^{2}+\left(F_{\lambda} /(\mathrm{S} / \mathrm{N})\right)^{2}}$, where systematic errors included both a calibration error and $K_{s}$ band photometric uncertainty. For MIPS24 observations at high signal-to-noise ratio $(\mathrm{S} / \mathrm{N})$ the error is dominated by uncertainties in calibration and photosphere estimation, and was estimated to be $6 \%$. At $70 \mu \mathrm{m}$, the error in flux density is a combination of calibration uncertainty and background noise. We assume systematic errors in the $70 \mu \mathrm{m}$ values of $15 \%$ of the observed flux. In addition to this, background noise was estimated from each of the images and folded into the total $70 \mu \mathrm{m}$ uncertainty calculation.

\section{RESULTS AND DISCUSSION}

Out of 634 targets observed with MIPS24, 29 met our criteria for identification as debris-disk candidates. Definitive identification of a debris-disk origin for the infrared excess emission will require follow-up observations. Candidates are listed by Hipparcos catalog number in Table 1 together with the spectral types and distances to the target stars as well as MIPS24 flux density, estimated photosphere emission, flux/photosphere ratio, the $\mathrm{S} / \mathrm{N}$ of the observations, and the $\mathrm{S} / \mathrm{N}$ of the excess emission, $\chi_{24}$. Candidates comprise $4.6 \%$ of the sample with excess emission that ranges from $20 \%$ to $60 \%$ of photosphere values. Two thirds of both the overall targets surveyed and those on the candidate list have spectral types $\mathrm{K}$ and later, indicating no decrease in detection frequency with later spectral type.

Plavchan et al. (2009) pointed out that an M dwarf spectroscopic binary companion to a $\mathrm{K}$ dwarf primary will produce an apparent MIPS24 excess at the $10 \%$ level. Six of the candidates in Table 1 are known to be spectroscopic binaries. They all have MIPS24 excesses greater than $20 \%$ and relative spectral types that would produce a diminished effect compared to that mentioned by Plavchan et al. (2009). Several close binaries do have $\mathrm{K}$ and $\mathrm{M}$ spectral types for the primary and secondary, however. We calculate that their companions contribute less than $10 \%$ of the measured $K-24$ color and cannot be considered as a sole source of an artifactual excess.

Out of 436 MIPS70 observations, only 145 stars were detected at $3 \sigma$ levels or greater. Of these, 21 stars yielded evidence of candidate debris disks and are listed in Table 2. Target and observational properties are given as in Table 1, together with an additional column as described below. As for MIPS24 observations, the distribution of spectral types is in agreement 
Table 1

Measured and Predicted Flux Densities (MIPS24)

\begin{tabular}{|c|c|c|c|c|c|c|c|}
\hline HIP & $S p T$ & $\begin{array}{c}\text { Distance } \\
(\mathrm{pc})\end{array}$ & $\begin{array}{c}F_{\text {MIPS24 }} \\
(\mathrm{mJy})\end{array}$ & $\begin{array}{c}F_{*} \\
(\mathrm{mJy})\end{array}$ & $F_{\text {MIPS24 }} / F_{*}$ & $\mathrm{~S} / \mathrm{N}$ & $\chi_{24}$ \\
\hline 169 & $\mathrm{~K} 5 \mathrm{~V}$ & 15.96 & 46.97 & 34.95 & 1.34 & 33.49 & 4.96 \\
\hline $171^{\mathrm{a}}$ & $\mathrm{G} 5 \mathrm{Vb}$ & 12.46 & 215.53 & 175.34 & 1.23 & 71.89 & 4.69 \\
\hline 3588 & K7V & 16.06 & 87.89 & 59.61 & 1.47 & 45.97 & 7.46 \\
\hline $3810^{\mathrm{a}}$ & F8V & 23.96 & 212.35 & 168.02 & 1.26 & 71.52 & 5.25 \\
\hline $5336^{\mathrm{a}}$ & $\mathrm{G} 5 \mathrm{Vb}$ & 7.66 & 343.01 & 279.78 & 1.23 & 90.75 & 4.25 \\
\hline $12623^{a}$ & F9V & 24.76 & 259.75 & 214.49 & 1.21 & 79.01 & 3.97 \\
\hline 13642 & G5V & 22.96 & 63.45 & 48.17 & 1.32 & 39.12 & 4.90 \\
\hline 17750 & M1.5V & 17.46 & 25.38 & 16.00 & 1.59 & 24.68 & 6.33 \\
\hline 21770 & F2V & 20.16 & 276.63 & 228.00 & 1.21 & 81.28 & 4.02 \\
\hline 24783 & $\mathrm{~K} 7 \mathrm{~V}$ & 20.36 & 34.60 & 26.55 & 1.30 & 28.78 & 4.27 \\
\hline 36827 & $\mathrm{~K} 2 \mathrm{~V}$ & 24.86 & 35.63 & 28.05 & 1.27 & 28.94 & 3.91 \\
\hline 44295 & $\mathrm{~K} 4 \mathrm{~V}$ & 18.36 & 52.99 & 32.19 & 1.65 & 35.52 & 7.76 \\
\hline 54952 & $\mathrm{~K} 5 \mathrm{~V}$ & 14.76 & 77.87 & 62.20 & 1.25 & 43.36 & 4.20 \\
\hline 58345 & K5V & 10.26 & 139.11 & 112.94 & 1.23 & 57.86 & 4.14 \\
\hline 58576 & G9IV & 12.96 & 214.13 & 172.15 & 1.24 & 71.77 & 4.43 \\
\hline $72848^{a}$ & $\mathrm{~K} 2 \mathrm{~V}$ & 11.56 & 200.82 & 133.19 & 1.51 & 69.33 & 7.58 \\
\hline 73633 & K3V & 24.36 & 32.05 & 26.55 & 1.21 & 27.66 & 3.09 \\
\hline 74975 & F8III-IV & 24.76 & 234.84 & 190.40 & 1.23 & 75.07 & 4.29 \\
\hline 77952 & F2III & 12.36 & 1256.26 & 947.00 & 1.33 & 173.49 & 5.81 \\
\hline 78734 & $\mathrm{~K} 8 \mathrm{Vk}$ & 23.36 & 20.91 & 17.00 & 1.23 & 22.56 & 3.06 \\
\hline 79492 & G8V & 24.46 & 82.53 & 60.57 & 1.36 & 43.57 & 5.56 \\
\hline 83101 & $\mathrm{~K} 5 \mathrm{~V}$ & 19.16 & 54.37 & 45.10 & 1.21 & 36.45 & 3.40 \\
\hline 85561 & K5V & 18.26 & 23.95 & 19.60 & 1.22 & 33.42 & 3.52 \\
\hline 86282 & $\mathrm{~K} 5 \mathrm{~V}^{\mathrm{b}}$ & 22.16 & 33.42 & 22.20 & 1.51 & 28.15 & 6.10 \\
\hline 99316 & $\mathrm{~K} 0 \mathrm{~V}$ & 24.36 & 51.07 & 40.47 & 1.26 & 34.91 & 4.08 \\
\hline 101150 & K5V & 23.16 & 26.33 & 20.30 & 1.30 & 25.25 & 3.97 \\
\hline 102488 & K0III & 22.16 & 5678.52 & 3770.00 & 1.51 & 368.49 & 7.99 \\
\hline 104239 & K1V & 17.66 & 87.26 & 70.78 & 1.23 & 45.64 & 3.99 \\
\hline $117712^{\mathrm{a}}$ & K3V & 10.86 & 212.55 & 157.08 & 1.35 & 71.24 & 5.89 \\
\hline
\end{tabular}

Notes.

${ }^{\text {a }}$ Spectroscopic binary.

${ }^{\mathrm{b}}$ Spectral type estimated from $T_{\mathrm{eff}}=4400 \mathrm{~K}$.

Table 2

Measured and Predicted Flux Densities (MIPS70)

\begin{tabular}{lccrrrrrr}
\hline \hline HIP & SpT & $\begin{array}{c}\text { Distance } \\
(\mathrm{pc})\end{array}$ & $\begin{array}{c}F_{\text {MIPS70 }} \\
(\mathrm{mJy})\end{array}$ & $\begin{array}{c}F_{*} \\
(\mathrm{mJy})\end{array}$ & $F_{\text {MIPS70 }} / F_{*}$ & $\mathrm{~S} / \mathrm{N}$ & $\chi_{70}$ & $f_{d}$ \\
\hline 1368 & K7 & 14.99 & 27.88 & 5.15 & 5.41 & 5.23 & 3.35 & $1.1 \times 10^{-4}$ \\
1499 & G0V & 23.44 & 64.45 & 8.61 & 7.48 & 8.66 & 4.58 & $7.1 \times 10^{-5}$ \\
1598 & G0 & 24.84 & 44.75 & 5.35 & 8.37 & 7.37 & 4.35 & $6.6 \times 10^{-5}$ \\
4148 & K3V & 14.08 & 30.25 & 8.77 & 3.45 & 5.48 & 3.01 & $3.8 \times 10^{-5}$ \\
8497 & F3III & 23.61 & 61.79 & 25.62 & 2.41 & 8.11 & 3.01 & $6.7 \times 10^{-6}$ \\
17439 & K0V & 16.23 & 83.30 & 8.45 & 9.85 & 10.05 & 4.99 & $1.1 \times 10^{-4}$ \\
19893 & F4III & 20.30 & 157.37 & 35.63 & 4.42 & 13.59 & 4.63 & $1.6 \times 10^{-5}$ \\
25775 & M0 & 19.39 & 28.22 & 2.74 & 10.30 & 5.91 & 3.99 & $2.4 \times 10^{-4}$ \\
30503 & G2IV & 22.04 & 110.75 & 8.93 & 12.40 & 11.12 & 5.26 & $1.0 \times 10^{-4}$ \\
32919 & K2 & 18.83 & 23.59 & 3.67 & 6.42 & 4.62 & 3.21 & $1.2 \times 10^{-4}$ \\
36827 & K2V & 24.80 & 45.63 & 3.09 & 14.79 & 6.77 & 4.43 & $1.9 \times 10^{-4}$ \\
43534 & K5 & 16.49 & 27.11 & 3.35 & 8.09 & 5.19 & 3.59 & $1.6 \times 10^{-4}$ \\
50384 & F8Vb & 22.72 & 42.93 & 13.01 & 3.30 & 6.99 & 3.36 & $1.9 \times 10^{-5}$ \\
58451 & K2V & 20.82 & 47.95 & 4.53 & 10.58 & 7.81 & 4.59 & $1.5 \times 10^{-4}$ \\
66781 & K0IV-V & 23.74 & 26.24 & 3.88 & 6.76 & 5.72 & 3.70 & $7.1 \times 10^{-5}$ \\
72387 & M2V & 24.24 & 16.91 & 2.14 & 7.90 & 4.35 & 3.18 & $1.6 \times 10^{-4}$ \\
88745 & F7V & 23.61 & 100.07 & 42.66 & 2.23 & 10.60 & 3.12 & $1.5 \times 10^{-5}$ \\
105184 & G2V & 23.79 & 106.27 & 6.25 & 17.01 & 11.31 & 5.41 & $1.4 \times 10^{-4}$ \\
108028 & K0 & 23.98 & 42.66 & 3.20 & 13.33 & 7.34 & 4.57 & $1.9 \times 10^{-4}$ \\
112190 & K0 & 21.52 & 22.37 & 3.88 & 5.76 & 5.35 & 3.45 & $7.4 \times 10^{-5}$ \\
117779 & K5 & 22.41 & 20.57 & 2.45 & 8.38 & 5.01 & 3.53 & $1.9 \times 10^{-4}$ \\
\hline & & & & & & &
\end{tabular}

with that for the general sample. In the overall sample (436 stars), $70 \%$ of the targets had spectral types $\mathrm{K}$ and later. The detected sample (145 stars) was biased in favor of early types, however, so less than half were $\mathrm{K}$ or later. Nevertheless, almost two thirds (62\%) of the MIPS70 debris-disk candidates have spectral types $\mathrm{K}$ and later. 
Targets in Table 2 are independent from those listed in Table 1 with the exception of a single K2V star, HIP 36827. A simplistic detection rate derived from the MIPS70 observed sample, $21 / 436=4.8 \%$, is lower than that of other surveys and probably reflects the influence of low sensitivity for distant faint targets. A rate derived only from objects detected at $3 \sigma$ levels, $21 / 145=14.5 \%$ is closer to results reported elsewhere in the literature. A better estimate of the detection rate requires modeling of the variable sensitivity for the entire sample with estimates of the upper limits of detection for infrared excess. This will be presented in a future manuscript together with Spitzer results for all 634 stars (S. Kim et al. 2010, in preparation).

MIPS70 excesses can be expressed as a lower limit to the fraction of stellar infrared luminosity that is absorbed and re-radiated by an optically thin debris disk, under the assumption that excess emission comes from a distribution of grains radiating as a $50 \mathrm{~K}$ blackbody with peak emission near $70 \mu \mathrm{m}$. Values of this expression, $f_{d} \sim L_{\mathrm{IR}} / L_{\mathrm{star}}$, are listed in the final column to Table 2 and range from $6.7 \times 10^{-6}$ to $2.4 \times 10^{-4}$. For comparison, $f_{d} \approx 9.7 \times 10^{-5}$ for $\epsilon$ Eri (Backman \& Paresce 1993). Twelve of the targets listed in Table 1 have lower-limit values of $f_{d}$ greater than this, suggesting that debris disks as massive as that around $\epsilon$ Eri are present at the $3 \%$ level or greater.

Tables 1 and 2 tally a total of 49 debris-disk candidates, since only one target is common to both lists. HIP 36827 exhibits excess infrared emission with flux densities of $7.6 \mathrm{mJy}$ and $42.5 \mathrm{mJy}$ at MIPS24 and MIPS70, respectively. The color temperature implied by these values is $T_{\text {col }}=80 \mathrm{~K}$ with an implied distance of $8 \mathrm{AU}$ for blackbody grains orbiting the K2 primary star.

The paucity of candidates with excess detected at both 24 and $70 \mu \mathrm{m}$ supports the idea of independent reservoirs of dust grains, a warm one that belongs to an inner planetary region and a colder "exo-Kuiper" Belt. Incongruence between targets in Tables 1 and 2 indicate that the presence of dust grains is unrelated between the regions. This notion accords well with the idea that the most massive debris disks are the result of larger collisions that occur periodically in time and independently between asteroidal and Kuiper Belt regions. The large number of giant stars in the MIPS24 sample may suggest an alternate explanation, such as the presence of a dust shell (see Jones 2008 and references therein). Refined understanding of dust source regions will require multi-wavelength observations at high angular resolution, as has been carried out for $\epsilon$ Eri (e.g., Backman et al. 2009).

We are grateful to I. Song and J. Rhee for use of their SED fitting software in analysis of our results. This work is based on observations made with the Spitzer Space Telescope, which is operated by the Jet Propulsion Laboratory, California Institute of Technology under a contract with NASA. Support for this work was provided by NASA through an award issued by JPL/Caltech.

\section{REFERENCES}

Backman, D., \& Paresce, F. 1993, in Protostars and Planets III, ed. E. H. Levy \& J. I. Lunine (Tucson, AZ: Univ. Arizona Press), 1253

Backman, D., et al. 2009, ApJ, 690, 1522

Beichman, C. A., et al. 2005, ApJ, 622, 116

Beichman, C. A., et al. 2006, ApJ, 652, 1674

Bryden, G., et al. 2006, ApJ, 636, 1098

Gautier, T. N., et al. 2007, ApJ, 667, 527

Hauschildt, P. H., Allard, F., \& Baron, E. 1999, ApJ, 512, 377

Jones, M. H. 2008, MNRAS, 387, 845

Plavchan, P., et al. 2009, ApJ, 698, 1068

Rieke, G. H., et al. 2004, ApJS, 154, 25

Rieke, G. H., et al. 2005, ApJ, 620, 1010

Rieke, G. H., et al. 2008, AJ, 135, 2245

Skrutskie, M. F., et al. 1997, in The Impact of Large Scale Near-IR Sky Surveys, ed. F. Garzon et al. (Kluwer: Dordrecht), 25

Su, K. Y. L., et al. 2006, ApJ, 653, 675

Trilling, D. E., et al. 2007, ApJ, 658, 1289

Trilling, D. E., et al. 2008, ApJ, 674, 1086

Wheelock, S., et al. 1993, http://irsa.ipac.caltech.edu/IRASdocs/issa.exp.sup/ index.html 